\title{
Study of nuclear structure effect on fusion through barrier distribution for the system ${ }^{28} \mathrm{Si}_{+}{ }^{154} \mathrm{Sm}$
}

\author{
Gurpreet Kaur $^{1, a}$, B.R. Behera ${ }^{1}$, A. Jhingan², P. Sugathan² ${ }^{2}$ and N. Rowley ${ }^{3}$ \\ ${ }^{1}$ Department of Physics, Panjab University, Chandigarh-160014, India \\ ${ }^{2}$ Inter University Accelerator Centre, New Delhi-110067, India \\ ${ }^{3}$ Institut de Physique Nucléaire, Orsay Cedex, France
}

\begin{abstract}
We plan to perform large angle quasi-elastic scattering experiments using a ${ }^{28} \mathrm{Si}$ beam on heavy targets in order to study the nuclear dynamics and structure effects. As a prelude to our first test experiment with a ${ }^{154} \mathrm{Sm}$ target, we study here the existing fusion data for the same system through the barrier distribution.
\end{abstract}

\section{Introduction}

In heavy ion reactions, coupling to different channels affects the fusion process and gives information about the nuclear properties through the fusion excitation function [1] and the barrier distribution (BD) [2]. Sometimes, even though a coupling appears to describe the excitation function quite well, it may fail to explain the BD. Moreover, the fusion excitation function calculations fail to reveal the structure effect on the higher energy side of the barrier. But, the transformation of fusion cross section to BD has an advantage of showing this effect over the entire range of energy. It has been suggested that channel couplings also affect the scattering process and the nuclear structure information can also be obtained from quasi-elastic (QE) scattering cross sections at large backward angles [3] and the corresponding BD [4]. Indeed this even allows the study of very heavy systems leading to the creation of super-heavy compound nuclei [5-6] where fission and quasi-fission dominate, and the "fusion" barrier distribution becomes meaningless. We ultimately wish to perform such experiments using a ${ }^{28} \mathrm{Si}$ beam on heavy targets.

In ref. [7], the authors obtained $\mathrm{BD}$ for the first time directly from experimental fusion cross section data, with $10 \%$ statistical error, on the systems ${ }^{16} \mathrm{O}+{ }^{154} \mathrm{Sm}$ and ${ }^{16} \mathrm{O}+{ }^{148} \mathrm{Sm}$. Although the data showed distributions significantly wider than the "one-dimensional barrier" case (no coupling), there was insufficient detail in them to allow one to extract information on the relevant target deformations. Wei et al. [8] for the first time studied the system ${ }^{16} \mathrm{O}+{ }^{154} \mathrm{Sm}$ through the BD with high precision data at small and well defined energy intervals and showed the clear visualization of rotational excitation of the target ${ }^{154} \mathrm{Sm}$. Similar kind of work has also been performed for the system ${ }^{16} \mathrm{O}+{ }^{148} \mathrm{Sm}$ and reported the vibration excitation of ${ }^{148} \mathrm{Sm}[9]$.

\footnotetext{
ae-mail: gur.1187@gmail.com
}

As stated above, we wish to perform experiments using a ${ }^{28} \mathrm{Si}$ beam on heavy targets. This projectile has a much larger charge than ${ }^{16} \mathrm{O}$ and this can magnify the effects of the coupling on BD structures due to dependence of coupling strength on the product of charges of the interacting nuclei. However, unlike ${ }^{16} \mathrm{O},{ }^{28} \mathrm{Si}$ almost certainly cannot be treated as inert, and this may introduce further structures into the BD rather than just widening it. Before performing the QE experiment, we shall study here the existing fusion data on the ${ }^{28} \mathrm{Si}+{ }^{154} \mathrm{Sm}$ system [10] to see what can be learned from them. Although these data were not taken with a view to deriving a BD, they will prove good enough to obtain a distribution against which we can compare our various calculations. Data for this system are available with an energy step of around $2 \mathrm{MeV}$, but the experimental error has not been reported. Similar experimental work [11] generally reports an error of around $10 \%$. So we assumed this value here and extracted the corresponding $\mathrm{BD}$. The extracted $\mathrm{BD}$ has been compared with that obtained from coupled-channel calculations using different coupling schemes for the target and projectile; the results will guide us in performing the necessary scattering calculations for our future QE work, where this system will be used for our first test experiment.

\section{Theoretical Approach}

\subsection{Preliminary Calculation}

Since the target ${ }^{154} \mathrm{Sm}$ falls into the category of a rotor, so as a preliminary calculation the effect of its deformation on the interaction potential has been studied. The potential has been calculated considering the line of approach of the projectile along the symmetry axis of the deformed target which is the lowest energy configuration. It is evident from figure 1 that there is a significant variation of fusion barrier with deformation of ${ }^{154} \mathrm{Sm}$. As the deformation parameter is increased from 0 to 0.3 , fusion barrier 


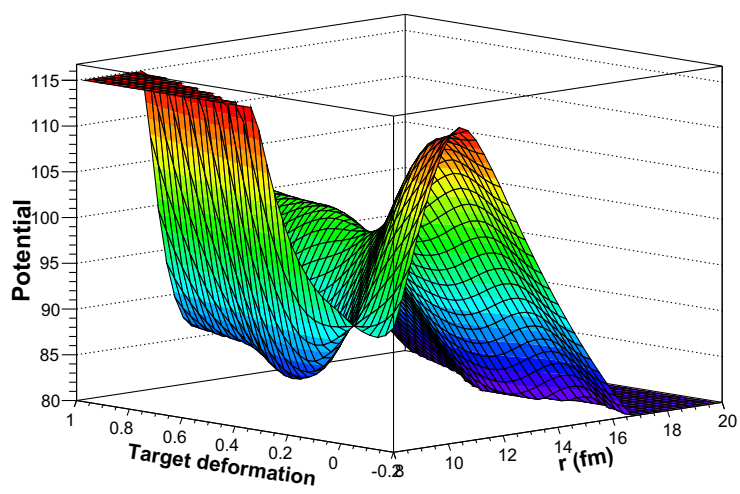

Figure 1. Variation of interaction potential between the nuclei as a function of inter-nuclear radial distance (r) and quadrupole deformation parameter of target, ${ }^{154} \mathrm{Sm}$.

decreases. Since deformation of the target shows a prominent effect on fusion barrier, we can expect the system to have a well-shaped BD. Also the excitation of the projectile, ${ }^{28} \mathrm{Si}$, may modify the distribution. So, in addition to the target deformation we also wish to incorporate the coupling of projectile excitation in our coupled channel calculations.

\subsection{Theoretical Calculations for BD}

From a theoretical point of view, the standard way to address the influence of coupling between the relative motion and the nuclear intrinsic degrees of freedom is through the use of the coupled-channels formalism. This includes couplings to static deformation, vibrational states and also transfer and breakup channels. In case of heavier nuclei, strength of the coupling is more and it is necessary to include higher-order terms in the expansion of coupling potential. Since heavy nuclei were involved in our system it is not advisable to limit this expansion to the linear term of the deformation parameter. Thus, CCFULL code [12] has been implemented here, instead of CCMOD [13], to get the theoretical fusion cross sections which treats the excitation energies of the coupled states correctly.

From experimental and calculated fusion cross section, the barrier distributions have been obtained by taking the second derivative of the product $E_{c . m} \sigma_{c . m}$, with respect to $E_{c . m}$, where $\sigma_{c . m}$ is the fusion cross section and $E_{c . m}$ is the energy in center of mass frame. Numerically this was calculated using a point difference formula. For data with equal energy steps, i.e., $\Delta E=\left(E_{2}-E_{1}\right)=\left(E_{3}-E_{2}\right)$, the expression at energy $E=\left(E_{1}+2 E_{2}+E_{3}\right) / 4$, is given by

$$
\frac{d^{2}(E \sigma)}{d E^{2}}=\frac{(E \sigma)_{3}-2(E \sigma)_{2}+(E \sigma)_{1}}{\Delta E^{2}}
$$

where $(\mathrm{E} \sigma)_{i}$ are evaluated at energies $E_{i}$. The statistical error $\delta_{c}$ associated with the second derivative at energy $E$ is approximately given by

$$
\delta_{c} \simeq\left(\frac{E}{\Delta E^{2}}\right)\left[(\delta \sigma)_{1}^{2}+4(\delta \sigma)_{2}^{2}+(\delta \sigma)_{3}^{2}\right]^{\frac{1}{2}}
$$

where $(\delta \sigma)_{i}$ are the absolute cross section uncertainties. The details about the method of extracting the BD has been reported in ref. [14].

\section{Results and discussion}

Assuming the statistical error of around $10 \%$ in the existing data for fusion cross section $(\sigma)$, the uncertainty in the $\mathrm{BD}$ has been calculated by using eq. (2). As the measurements were made with the tandem accelerator, so the error in the energy is assumed to be small enough to neglect. The nuclear potential in the coupled channel calculations has been described as the Woods-Saxon potential defined by $V_{0}, r_{0}$ and $a_{0}$; where $V_{0}$ is the depth parameter, $r_{0}$ is the radius parameter, and $a_{0}$ is the surface diffuseness parameter. These parameters were obtained by fitting the experimental excitation function above the barrier as this high energy part is less sensitive to the coupling effects. The obtained value of parameters are $V_{0}=150 \mathrm{MeV}$, $r_{0}=1.10 \mathrm{fm}$ and $a_{0}=0.72 \mathrm{fm}$ which give an uncoupled barrier height of $102.2 \mathrm{MeV}$, close to the Bass value (102.9 $\mathrm{MeV}$ ). The comparison of experimental BD with that obtained from coupled channel calculation has been shown in figure 2. The BD without any coupling has large deviation from experimental $\mathrm{BD}$ as shown in figure 3 with $[(0,0) ; 0]$.

In figure 2 and 3 , we will be using the notation $\left[\left(n_{1}, n_{2}\right) ; n_{3}\right]$ to describe a state of the interacting nuclei. Here, $n_{1}$ and $n_{2}$ denote the number of quadrupole and octupole vibrational excitation of ${ }^{28} \mathrm{Si}$ projectile, respectively, whereas $n_{3}$ represents the number of rotational states of ${ }^{154} \mathrm{Sm}$ nuclei included in the coupled channel calculation.

Since in the case of ${ }^{16} \mathrm{O}+{ }^{154} \mathrm{Sm}$, it was observed that coupled channel calculation involving only the rotational deformation of the target ${ }^{154} \mathrm{Sm}$ was explaining the experimental BD [8], we have resorted to similar type of calculations for our system taking ${ }^{28} \mathrm{Si}$ as inert. We considered up to $6^{+}$rotational states of the ${ }^{154} \mathrm{Sm}$ target with $\left[(0,0) ; n_{3}\right]$ where, $n_{3}$ varies from 1 to 3 , i.e., three rotational states were excited. The quadrupole deformation parameter $\beta_{2}$ as 0.341 , octupole deformation parameter $\beta_{4}$ as 0.07 with excitation energy of $2^{+}$state as $0.08197 \mathrm{MeV}$ have been used. The corresponding distributions are shown in figure 2(a) and 2(b). In addition to this, different couplings of vibrational excitations in ${ }^{28} \mathrm{Si}$ have been considered using the quadrupole deformation parameter $\left(\beta_{2}\right)$ as -0.407 with excitation energy of $2^{+}$state as $1.78 \mathrm{MeV}$. As shown in figure 2(c) to 2(f), the BD has different shapes when these are taken into account and good experimental data could, in principle, distinguish between them. However, the present data were not taken with sufficient precision to do this. It has been observed that the assumption of an inert projectile, as made in the case of ${ }^{16} \mathrm{O}$, is not adequate here but we need better data to explore this. The effect of adding the octupole phonon excitation of the target is also considered and is shown in figure 2(e).

In figure 3 , the comparison of experimental and theoretical fusion cross section and the corresponding BD is 
(a)

(b)

(c)

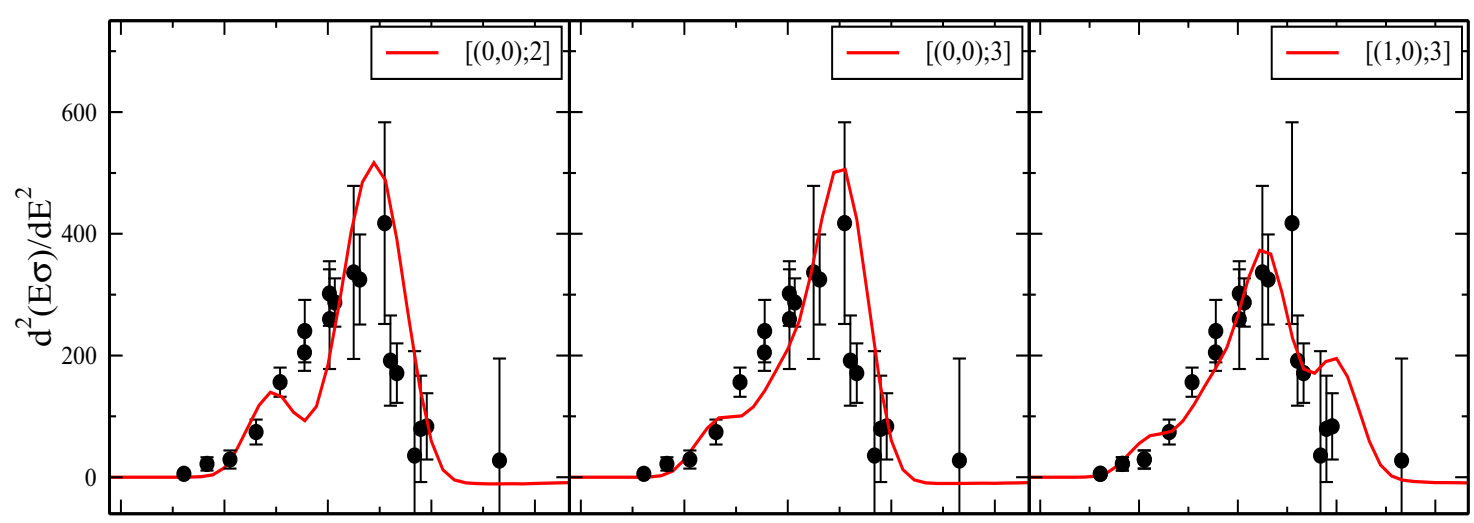

(d)

(e)

(f)

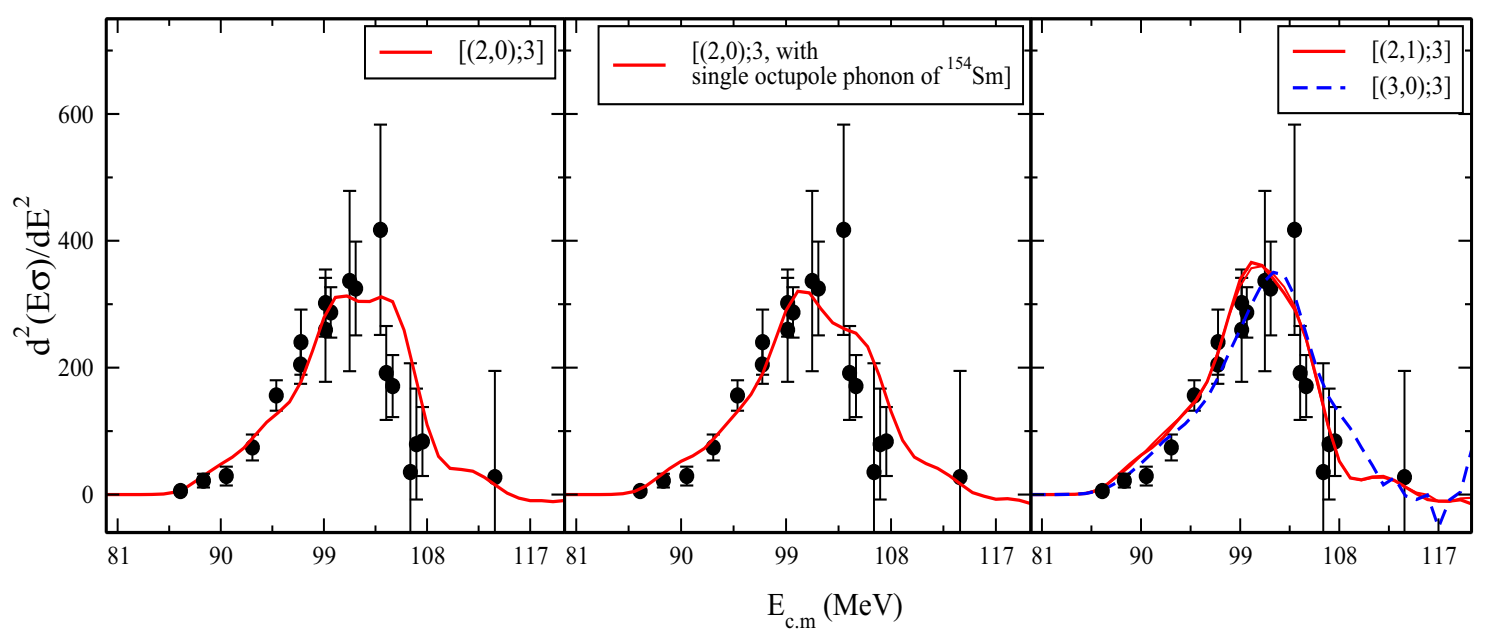

Figure 2. Barrier distributions obtained from coupled channel calculations with different excitation of target ${ }^{154} \mathrm{Sm}$ and projectile ${ }^{28} \mathrm{Si}$. The notation $\left[\left(n_{1}, n_{2}\right) ; n_{3}\right]$ gives the excitation included in the corresponding part of figure and its description is given in the text. Dots are the experimental barrier distribution.
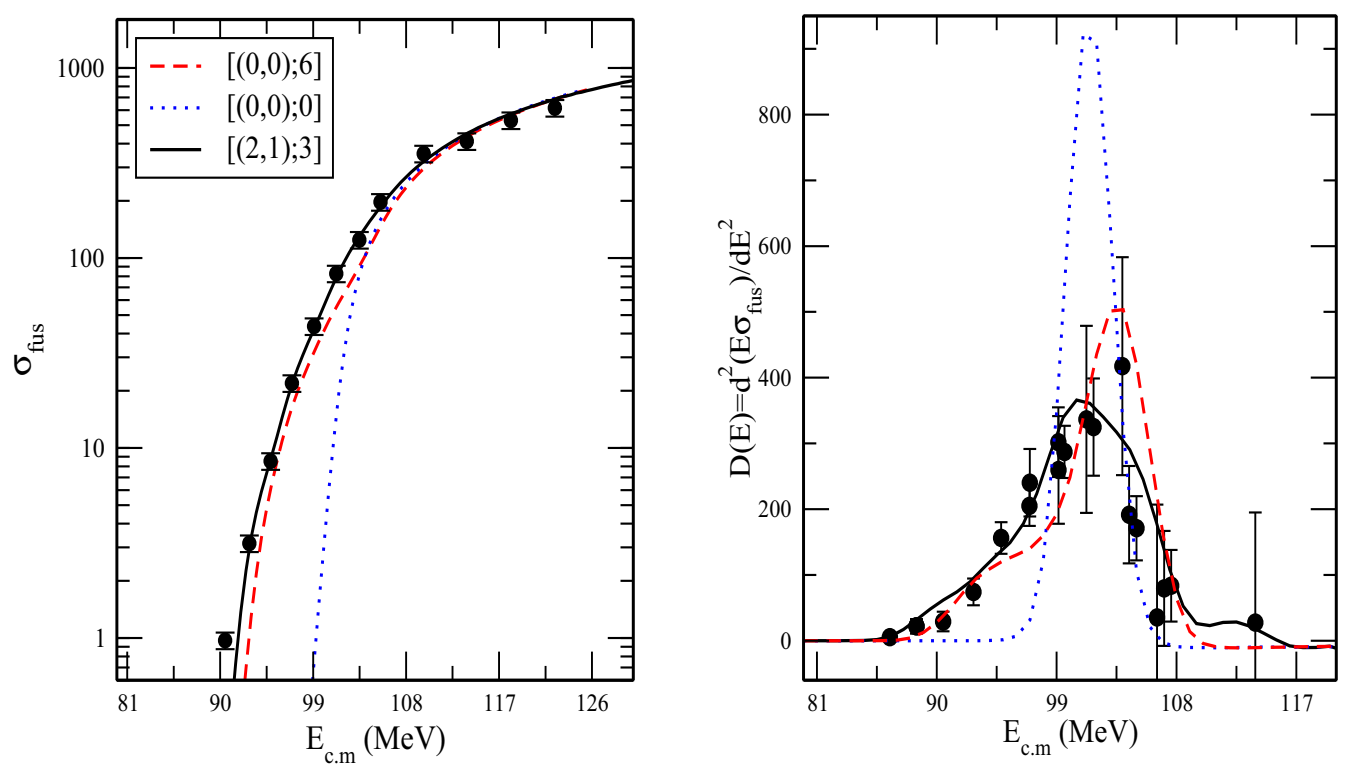

Figure 3. Fusion cross section (left) and barrier distributions (right) for ${ }^{28} \mathrm{Si}+{ }^{154} \mathrm{Sm}$. Dots are the experimental data, dotted line for without coupling, dashed and solid lines for coupling mentioned inside figure. The notation $\left[\left(n_{1}, n_{2}\right) ; n_{3}\right]$ gives the excitation included and its description is given in the text. 
shown. Although little difference is observed in the excitation function due to different coupling but this difference is clearly visible in the BD calculation. The width of the distribution is observed to be larger than that of uncoupled BD. Since the width of the BD denotes the coupling strength which is consistent with the coupling during the interaction.

In the ref. [10], it has been reported that the octupole vibration of ${ }^{154} \mathrm{Sm}$, in addition to its static deformation and quadrupole vibration of ${ }^{28} \mathrm{Si}$, is explaining the experimentally observed fusion excitation function for the system ${ }^{28} \mathrm{Si}+{ }^{154} \mathrm{Sm}$. But here, through the BD calculations we have observed that within the experimental error bars the octupole vibration of both ${ }^{28} \mathrm{Si}$ and ${ }^{154} \mathrm{Sm}$ are seen to be showing almost similar effect. The Q-value for neutron transfer channels in this system is positive but they have not been considered in the present work.

\section{Summary and Conclusions}

To study the possible role of the structure of ${ }^{28} \mathrm{Si}$, we have obtained an approximate fusion BD for the system ${ }^{28} \mathrm{Si}$ $+{ }^{154} \mathrm{Sm}$ from the existing data despite the fact that the data were not taken with the precision of Wei et al [8]. It has been observed that in addition to the static deformation of permanently deformed target ${ }^{154} \mathrm{Sm}$, the dynamic deformation of projectile ${ }^{28} \mathrm{Si}$ could not be ignored for explaining the fusion dynamics. Whereas for the system ${ }^{16} \mathrm{O}$ $+{ }^{154} \mathrm{Sm}$, BD were well described by considering only the rotational excitation of ${ }^{154} \mathrm{Sm}$. Thus, showing the effect of projectile excitation during the fusion process.

Unfortunately, the precision of the experimental data impedes us from deep understanding the nuclear structure responsible for the fusion process, i.e., whether octupole or double quadrupole excitation of ${ }^{28} \mathrm{Si}$ nuclei or octupole excitation of ${ }^{154} \mathrm{Sm}$ is responsible for the observed fusion process. Because from these cases it is difficult to resolve the structures of the BD for best fit as the error bars are too large. The deciding factor to resolve these nuclear structures would be the experimental precision at the higher energy side of the fusion barrier. Need for high precision data can be fulfilled by performing either fusion or QE measurement. As for heavy systems, QE is more appropriate so we intend to perform the QE measurement for this system in the future and we hope that our first QE experiment might shed some light on this.

Since our present version of the CCFULL program does not permit rotor-plus-rotor calculations, we have simply treated the ${ }^{28} \mathrm{Si}$ nucleus as a harmonic vibrator. However, elsewhere, we shall relax this condition and study this system with more generalized couplings.

\section{Acknowledgments}

The authors are grateful to Professor K. Hagino, Professor M. Dasgupta and Dr. B.K. Nayak for stimulating dis- cussions. One of the authors, G. K. acknowledges the financial assistance from the University Grants Commission (UGC), New Delhi under the Maulana Azad National Fellowship (MANF) for this work.

\section{References}

[1] S.G. Steadman and M.J. Rhoades-Brown, Annu. Rev. Nucl. Part. Sci. 36, 649 (1986)

[2] M. Dasgupta, D.J. Hinde, N. Rowley, and A.M. Stefanini, Annu. Rev. Nucl. Part. Sci. 48, 401 (1998)

[3] M.V. Andres, N. Rowley, and M.A. Nagarajan, Phys. Lett. B 202, 292 (1988)

[4] H. Timmers, J.R. Leigh, M. Dasgupta, D.J. Hinde, R.C. Lemmon, J.C. Mein, C.R. Morton, J.O. Newton, and N. Rowley, Nucl. Phys. A 584, 190 (1995)

[5] S. Mitsuoka, H. Ikezoe, K. Nishio, K. Tsuruta, S.C. Jeong, and Y. Watanabe, Phys. Rev. Lett. 99, 182701 (2007)

[6] S.S. Ntshangase N. Rowley, R.A. Bark, S.V. Förtsch, J.J. Lawrie, E.A. Lawrie, R. Lindsay, M. Lipoglavsek, S.M. Maliage, L.J. Mudau, S.M. Mullins, O.M. Ndwandwe, R. Neveling, G. Sletten, F.D. Smit, and C. Theron, Phys. Lett. B 651, 27 (2007)

[7] N. Rowley, G.R. Satchler, and P.H. Stelson, Phys. Lett. B 254, 25 (1991)

[8] J.X. Wei, J.R. Leigh, D.J. Hinde, J.O. Newton, R.C. Lemmon, S. Elfstrom, J.X. Chen, and N. Rowley, Phys. Rev. Lett. 67, 3368 (1991)

[9] J.R. Leigh, M. Dasgupta, D.J. Hinde, J.C. Mein, C.R. Morton, R.C. Lemmon, J.P. Lestone, J.O. Newton, H. Timmers, J.X. Wei, and N. Rowley, Phys.Rev. 52, 3151 (1995)

[10] S. Gil, D. Abriola, D.E. DiGregorio, M. di Tada, M. Elgue, A. Etchegoyen, M.C. Etchegoyen, J. Fernández Niello, A.M.J. Ferrero, A.O. Macchiavelli, A.J. Pacheco, J.E. Testoni, P. Silveira Gomes, V.R. Vanin, A. Charlop, A. García, S. Kailas, S.J. Luke, E. Renshaw, and R. Vandenbosch, Phys. Rev. Lett. 65, 3100 (1990)

[11] R.G. Stokstad, Y. Eisen, S. Kaplanis, D. Pelte, U. Smilansky, and I. Tserruya, Phys. Rev. Lett. 41, 465 (1978)

[12] K. Hagino, N. Rowley, and A.T. Kruppa, Comput. Phys. Commun. 123, 143 (1999)

[13] M. Dasgupta, A. Navin, Y.K. Agarwal, C.V.K. Baba, H.C. Jain, M.L. Jhingan, and A. Roy, Phys. Rev. Lett. 66, 1414 (1991)

[14] N. Rowley, Nucl. Phys. A 538, 205 (1992) 